\title{
SYSTEMATICS OF PROTON EMISSION
}

\author{
D.S. Delion \\ National Institute of Physics and Nuclear Engineering, \\ POB MG-6, Bucharest-Măgurele, Romania \\ R.J. Liotta and R. Wyss \\ KTH, Alba Nova University Center, SE-10691 Stockholm, Sweden
}

(Dated: August 21, 2018)

\begin{abstract}
A very simple formula is presented that relates the logarithm of the half-life, corrected by the centrifugal barrier, with the Coulomb parameter in proton decay processes. The corresponding experimental data lie on two straight lines which appear as a result of a sudden change in the nuclear shape marking two regions of deformation independently of the angular momentum of the outgoing proton. This feature provides a powerful tool to assign experimentally quantum numbers in proton emitters.
\end{abstract}

PACS numbers: 21.10.Tg, 23.50.+z, 24.10.Eq

Keywords: Proton emission, Half-life, Coulomb function, Angular momentum

Nearly a century ago one of the most challenging problems in physics was the understanding of the Geiger-Nuttall rule, which says that the logarithm of the half-life in alpha decay is inverse proportional to the energy of the outgoing alpha particle, i. e. its Q-value. It can be asserted that the probabilistic interpretation of quantum mechanics started by the explanation given by Gamow to that rule as a consequence of the quantum penetration of the alpha particle through the Coulomb barrier [1]. Nowadays the challenge in nuclear physics is related to rare nuclei, i. e. nuclei lying very far from the stability line which decay rapidly by particle emission (neutron and proton drip lines) [2]. The exploration of the drip-lines is one of the ambitions of the projected radioactive nuclear beam facilities as e. g. the Rare Isotope Accelerator (RIA) [3].

By plotting the logarithm of the half-lives as a function of $Q_{p}$, i. e. the kinetic energy of the emitted proton, one does not obtain a clear graphical pattern of the experimental data, as for the Geiger-Nuttall rule. As seen in Figure 1.a [4], such a plot does not reveal any obvious trend. The reason of this disorder is that not only $Q_{p}$ but also the height and width of the Coulomb and centrifugal barriers, in which the proton is trapped before decaying, determine the decay width. The role of the centrifugal barrier in proton emission is more important than in $\alpha$-decay due to the smaller proton reduced mass and also because in most cases the proton carries a non-vanishing angular momentum. The half-life corresponding to the wave with given quantum numbers $(l, j)$ can be written as $[\underline{5}$

$$
T_{1 / 2}=\frac{\ln 2}{v}\left|\frac{H_{l}^{(+)}(\chi, \rho)}{s_{l j}(R, \beta)}\right|^{2},
$$

where $H_{l}^{(+)}$is the Coulomb-Hankel spherical wave, $\chi=2(Z-1) e^{2} /(\hbar v)$ is the Coulomb parameter (which determines the Coulomb barrier), $Z$ is the charge number of the mother nucleus, $v=\hbar k / \mu=\sqrt{2 Q_{p} / \mu}$ is the velocity of the outgoing proton, $\mu$ is the reduced mass of the proton-daughter system, $\rho=k R$ and the spectroscopic function is given by $[\underline{6}]$

$$
s_{l j}(R, \beta)=\sum_{l^{\prime} j^{\prime}} K_{l j ; l^{\prime} j^{\prime}}(R, \beta) s_{l^{\prime} j^{\prime}}^{(0)} f_{l^{\prime} j^{\prime}}(R, \beta),
$$

where $s_{l j}^{(0)}$ is the so-called spectroscopic factor (the particle amplitude $u_{l j}$ within the super fluid model) and $f_{l j}$ are the components of the internal wave function (the standard Nilsson function within the adiabatic approach). We mention here that $\left|s_{l j}^{(0)}\right|^{2} \approx 0.5$. Moreover for spherical emitters it was evidenced a clear dependence of the spectroscopic factor upon the shell occupancy [7]. The asymptotic propagator matrix is given by

$$
K_{l j ; l^{\prime} j^{\prime}}(R, \beta) \equiv H_{l}^{(+)}(\chi, \rho)\left[\mathcal{H}^{(+)}(R, \beta)\right]_{l j ; l^{\prime} j^{\prime}}^{-1}=\delta_{l l^{\prime}} \delta_{j j^{\prime}}+\Delta K_{l j ; l^{\prime} j^{\prime}}(R, \beta) .
$$

Here $\mathcal{H}_{l j ; l^{\prime} j^{\prime}}^{(+)}(R, \beta)$ is the matrix of solutions with an outgoing boundary behavior, i.e. $\mathcal{H}_{l j ; l^{\prime} j^{\prime}}^{(+)}(R, \beta) \rightarrow_{R \rightarrow \infty} \delta_{l l^{\prime}} \delta_{j j^{\prime}} H_{l}^{(+)}(\chi, \rho)$. Thus, the relation (10) has the same form as in the spherical 
case, but for deformed systems the spectroscopic function is a superposition of the different channel components $f_{l j}$.

The ratio $H_{l}^{(+)} / s_{l j}$, entering Eq. (1) (which is just the inverse of the scattering amplitude in the $(l, j)$ channel) does not depend upon the radius and this is an important test of accuracy in any coupled channels scheme. A good approximation of the function $H_{l}^{(+)}$for physical situations is given by the WKB value, i. e. [5]

$$
H_{l}^{(+)}(\chi, \rho) \approx G_{l}(\chi, \rho) \approx C_{l}(\chi, \rho)(\operatorname{ctg} \alpha)^{1 / 2} \exp [\chi(\alpha-\sin \alpha \cos \alpha)]
$$

where $G_{l}$ is the irregular Coulomb function. The influence of the centrifugal barrier is fully contained in the function $C_{l}$, which is given by

$$
C_{l}(\chi, \rho)=\exp \left[\frac{l(l+1)}{\chi} \operatorname{tg} \alpha\right], \quad \cos ^{2} \alpha=\frac{Q_{p}}{V_{c}(R)}=\frac{\rho}{\chi},
$$

$V_{c}(R)$ is the Coulomb potential at distance $R$. We will choose this distance as the matching radius, for which we will adopt the standard form, i. e. $R=1.2\left(A_{d}^{1 / 3}+A_{p}^{1 / 3}\right)$, where $A_{d}$ is the mass number of the daughter nucleus and $A_{p}=1$. The dependence of the centrifugal factor upon the distance, entering through $\alpha$, is very weak around that value of $\mathrm{R}$. Defining a reduced half-life as

$$
T_{\text {red }}=\frac{T_{1 / 2}}{C_{l}^{2}}=\frac{F(\chi, \rho)}{\left|s_{l j}(R, \beta)\right|^{2}},
$$

where

$$
F(\chi, \rho)=\frac{\ln 2}{v} \operatorname{ctg} \alpha \exp [2 \chi(\alpha-\sin \alpha \cos \alpha)],
$$

one sees that $T_{\text {red }}$ should not depend upon the angular momentum $l$ if plotted against the dimensionless Coulomb parameter $\chi$. Moreover, $\log _{10} T_{\text {red }}$ is a linear function of $\chi$ independently of the value of $l$, provided the velocity $v$, the function $s_{l j}$ and the parameter $\alpha$, which depend upon the Q-value, has a smooth behavior in this logarithmic scale. Notice that the value of $T_{r e d}$ is model independent since the only theoretical quantity entering in its definition is the function $C_{l}^{2}$, which is a by-product of the barrier penetration process. But it is important to stress that this entire analysis is based upon the assumption that the proper value of $l$, that is the one that determines the experimental half-life $T_{1 / 2}$, is used. Otherwise, and since $T_{1 / 2}$ is strongly $l$-dependent, that straight line pattern would be completely spoiled.

To check the rather straightforward conclusions reached above we evaluated $\log _{10} T_{\text {red }}$ in cases where experimental data are available, as shown in Table 1 . We considered proton emitters with $Z>50$ and angular momentum as given in Ref. 4]. The orbital and total angular momenta of the proton in the mother nucleus labeling a given partial wave will be denoted by $\left(l_{m}, j_{m}\right)$. The corresponding outgoing values (at large distances) will be $(l, j)$. Angular momentum conservation requires that $\vec{J}_{m}=\vec{J}_{d}+\vec{j}$, where $J_{m}\left(J_{d}\right)$ is the angular momentum of the mother (daughter) nucleus. In the case of odd decaying nuclei to the ground state with $J_{d}=0$, it follows that $J_{m}=j_{m}$. If, in addition, the nucleus is spherical then $j_{m}=j$ and $l_{m}=l$, where $l_{m}$ is the orbital angular momentum of the single quasi-proton state in the mother nucleus. The value of $j_{m}$ in Table 1 is the angular momentum of the proton outside the nucleus. It is often extracted from theoretical predictions.

With the values of $l, \chi$ and $\log _{10} T_{\text {red }}$ of Table 1 we produced the plot shown in Figure 1.b. Amazingly enough, the points lie all approximately along two straight lines. The nuclei on the upper line correspond to the first twelve cases in Table 1 , i. e. those with $Z<68$. It is important to point out that the angular momenta in all cases of Figure 1.b are as reported in the literature, except the nucleus ${ }^{109} \mathrm{I}$ for which we changed the angular momentum $l=0$ of Ref. [4] to $l=2$. We did this because we performed a coupled channels calculation showing a larger component with $\mathrm{l}=2(0.37)$ in comparison with $\mathrm{l}=0(0.02)$ for the $1 / 2+$ deformed state at the Fermi level. Note that this assignment is also in agreement with the systematics in heavier isotopes, like e.g. in ${ }^{111} \mathrm{I}[8]$.

Using a fitting procedure we found that the experimental half-lives can be reproduced by the formula

$$
\begin{aligned}
\log _{10} T_{r e d}^{(k)} & =a_{k}(\chi-20)+b_{k}, \\
a_{1} & =1.31, \quad b_{1}=-2.44, \quad Z<68 \\
a_{2} & =1.25, \quad b_{2}=-4.71, \quad Z>68 .
\end{aligned}
$$


where $k=1$ corresponds to the upper line in Figure 1.b. The standard errors are $\sigma_{1}=0.26$ and $\sigma_{2}=0.23$, corresponding to a mean factor less than two.

These two straight lines may have been induced either by an abrupt change in the Q-values or in the structure of the different emitters, or by both. The Q-value dependence affects only the function $F(\chi, \rho)$. We therefore plotted this function against $\chi$ in Figure 2. One sees in this Figure the same pattern as in Figure 1.b, namely there appear two lines, but now the upper line includes only six emitters with $Z<68$. In fact the deviations from the two lines in Figure 2 are much larger here than in Figure 1.b, corresponding to an error of about one order of magnitude. This forces us to conclude that, although the pattern shown in Figure 2 corresponding to $s_{l j}(\beta)=$ const is similar to the dependence between $\rho$ and $\chi$, this effect can not be the only reason behind the two straight lines of Figure 1.b. The only other source that may contribute to that alignment is an abrupt change in the nuclear structure of the emitters. We therefore correlated the deformation values calculated in Ref. [11] with the determined half-lives. In Figure 3.a we show the deformation parameter $\beta$ as a function of $\mathrm{Z}$. At the proton drip line between $\mathrm{Z}=67$ and $\mathrm{Z}=69$ occurs a pronounced change, from a large prolate shape with $\beta \approx 0.3$ to an oblate shape with $\beta \approx-0.2$. These shapes are substantiated by measurements of moments of inertia. In order to analyze the dependence of the half-life upon the internal structure of the nucleus we plot in Figure 3.b the quantity

$\log _{10} s_{l j}^{-2}$ as a function of the charge number Z. It is worthwhile to emphasize once again that this is a model independent function.

One remarks a striking correlation between Figures 3.a and 3.b. After the jump occurring at $\mathrm{Z}=68$, where an abrupt shape change occurs, the deformation of the nuclei lying on the lower line smoothly increases. One can then assert that these lines reflect two regions of nuclei separated by a sharp transition between the prolate and oblate regimes.

There are two points in Figure 1.b that deviate conspicuously from the upper straight line. They correspond to ${ }^{140} \mathrm{Ho}(\mathrm{l}=3)$ and ${ }^{141} \mathrm{Ho}^{*}(\mathrm{l}=0)$. In Figure 3.a one sees that these nuclei are situated at the border between the two regions of deformation.

In conclusion we have presented in this paper a simple formula for proton decay (Eq. (8) ) similar to the Geiger-Nuttall rule. This formula enables the precise assignment of spin and parity for proton decaying states. The only quantities that are needed are the half-life of the mother nucleus and the proton Q-value. As a function of these quantities, corrected by the centrifugal barrier (Eq. (66)), the experimental data of proton emitters with $Z>50$ lie along two straight lines. Taking into account that ${ }^{140} \mathrm{Ho}^{141} \mathrm{Ho}^{*}$ are the only emitters that deviates from the systematics, one concludes the remarkable fact: proton emitters with $Z>50$ follow simple systematics, which are obviously correlated with two regions of quadrupole deformation, as can be seen by plotting $\log _{10} s^{-2}$ versus $\beta$.

\section{Acknowledgments}

This work has been supported by the Göran Gustafsson Foundation.

[1] G. Gamow, Z. f. Phys. 51, 204 (1928).

[2] P. J. Woods and C. N. Davids, Ann. Rev. Nucl. Part. Sci. 47, 541 (1997).

[3] Rare isotope accelerator. Argonne web page, www.anl.gov/ria, and Rare isotope accelerator (RIA). NSCL web page, www.nscl.msu.edu/ria

[4] A. A. Sonzogni, Nucl. Data Sheets 95, 1 (2002).

[5] P. O. Fröman, Mat. Fys. Skr. Dan. Vid. Selsk. 1, no. 3 (1957).

[6] D. S. Delion, R. J. Liotta, and R. Wyss, Phys. Rep. (2006) (in press).

[7] S. Åberg, P. B. Semmes and W. Nazarewicz, Phys. Rev. C 56, 1762 (1997).

[8] E. S. Paul et.al., Phys. Rev. C 61, 064320 (2000).

[9] P. J. Woods, et.al., Phys. Rev. C 69, 051302(R) (2004).

[10] A. P. Robinson, et.al., Phys. Rev. Lett. 95, 032502 (2005).

[11] P. Möller, R. J. Nix, W. D. Myers, and W. Swiatecki, At. Data Nucl. Data Tables 66, 131 (1995). 
Table 1

Data used in the calculation. The spin of the proton moving inside the mother nucleus $\left(j_{m}\right)$, the proton $Q$-value $\left(Q_{p}\right)$ and the emitter half-life $\left(T_{\text {exp }}\right)$ are from Refs. [4, , , 10]. The quadrupole deformation parameters $\beta$ are from Ref. [11] and the quantities $l, \chi, \alpha, T_{\text {red }}$ and $s_{l j}$ are as explained in the text. All half-lives are in seconds. Stars in the emitters indicate excited states.

\begin{tabular}{|c|c|c|c|c|c|c|c|c|c|c|}
\hline $\mathrm{No}$ & Emitter & $l$ & $j_{m}^{\pi}$ & $\begin{array}{c}Q_{p} \\
(\mathrm{keV})\end{array}$ & $\chi$ & $\beta$ & $\begin{array}{c}\alpha \\
(\mathrm{rad}) \\
\end{array}$ & $\log _{10} T_{\exp }$ & $\log _{10} T_{r e d}$ & $\log _{10} s_{l j}^{-2}$ \\
\hline 1 & ${ }_{51}^{105} \mathrm{Sb}$ & 2 & $5 / 2^{+}$ & $491(15)$ & 22.457 & 0.081 & 1.353 & 2.049 & 1.000 & 1.525 \\
\hline 2 & ${ }_{53}^{109} \mathrm{I}$ & 2 & $3 / 2^{+}$ & $829(3)$ & 17.977 & 0.160 & 1.290 & -3.987 & -4.994 & 1.810 \\
\hline 3 & ${ }_{55}^{112} \mathrm{Cs}$ & 2 & $3 / 2^{+}$ & $824(7)$ & 18.728 & 0.208 & 1.296 & -3.301 & -4.287 & 1.701 \\
\hline 4 & ${ }_{55}^{113} \mathrm{Cs}$ & 2 & $3 / 2^{+}$ & $978(3)$ & 17.191 & 0.207 & 1.270 & -4.777 & -5.747 & 1.213 \\
\hline 5 & ${ }_{57}^{117} \mathrm{La}$ & 2 & $3 / 2^{+}$ & $823(5)$ & 19.437 & 0.290 & 7.052 & -1.602 & -2.565 & 2.680 \\
\hline 6 & ${ }_{59}^{121} \mathrm{Pr}$ & 2 & $3 / 2^{+}$ & $900(10)$ & 19.253 & 0.318 & 7.119 & -2.000 & -2.939 & 2.756 \\
\hline 7 & ${ }_{63}^{130} \mathrm{Eu}$ & 2 & $3 / 2^{+}$ & $1028(15)$ & 19.263 & 0.331 & 7.263 & -3.046 & -3.941 & 2.146 \\
\hline 8 & ${ }_{63}^{131} \mathrm{Eu}$ & 2 & $3 / 2^{+}$ & $951(7)$ & 20.028 & 0.331 & 1.289 & -1.575 & -2.473 & 2.592 \\
\hline 9 & ${ }_{65}^{135} \mathrm{~Tb}$ & 3 & $7 / 2^{-}$ & $1188(7)$ & 18.499 & 0.325 & 7.341 & -3.027 & -4.770 & 2.548 \\
\hline 10 & ${ }_{67}^{140} \mathrm{Ho}$ & 3 & $7 / 2^{-}$ & $1106(10)$ & 19.775 & 0.297 & 7.416 & -2.222 & -3.937 & 1.864 \\
\hline 11 & ${ }_{67}^{141} \mathrm{Ho}$ & 3 & $7 / 2^{-}$ & $1190(8)$ & 19.064 & 0.286 & 1.261 & -2.387 & -4.094 & 2.672 \\
\hline 12 & ${ }_{67}^{141} \mathrm{Ho}^{*}$ & 0 & $1 / 2^{+}$ & $1256(8)$ & 18.557 & 0.286 & 1.252 & -5.180 & -5.180 & 2.268 \\
\hline 13 & ${ }_{69}^{145} \mathrm{Tm}$ & 5 & $11 / 2^{-}$ & $1753(10)$ & 16.185 & -0.199 & 7.490 & -5.409 & -9.500 & 1.307 \\
\hline 14 & ${ }_{69}^{146} \mathrm{Tm}$ & 5 & $11 / 2^{-}$ & $1210(5)$ & 19.481 & -0.199 & 1.261 & -1.276 & -5.460 & 0.943 \\
\hline 15 & ${ }_{69}^{146} \mathrm{Tm}^{*}$ & 5 & $11 / 2^{-}$ & $1148(5)$ & 20.001 & -0.199 & 1.270 & -0.456 & -4.651 & 1.053 \\
\hline 16 & ${ }_{69}^{147} \mathrm{Tm}$ & 5 & $11 / 2^{-}$ & $1071(3)$ & 20.708 & -0.190 & 1.280 & 0.591 & -3.613 & 1.148 \\
\hline 17 & ${ }_{69}^{147} \mathrm{Tm}^{*}$ & 2 & $3 / 2^{+}$ & $1139(5)$ & 20.080 & -0.190 & 1.271 & -3.444 & -4.282 & 1.324 \\
\hline 18 & ${ }_{71}^{150} \mathrm{Lu}$ & 5 & $11 / 2^{-}$ & $1283(4)$ & 19.477 & -0.164 & 1.255 & -1.180 & -5.280 & 1.314 \\
\hline 19 & ${ }_{71}^{150} \mathrm{Lu}^{*}$ & 2 & $3 / 2^{+}$ & $1317(15)$ & 19.224 & -0.164 & 1.251 & -4.523 & -5.342 & 1.592 \\
\hline 20 & ${ }_{71}^{151} \mathrm{Lu}$ & 5 & $11 / 2^{-}$ & $1255(3)$ & 19.694 & -0.156 & 1.259 & -0.896 & -4.996 & 1.316 \\
\hline 21 & ${ }_{71}^{151} \mathrm{Lu}^{*}$ & 2 & $3 / 2^{+}$ & $1332(10)$ & 19.116 & -0.156 & 1.249 & -4.796 & -5.613 & 1.475 \\
\hline 22 & ${ }_{73}^{155} \mathrm{Ta}$ & 5 & $11 / 2^{-}$ & $1791(10)$ & 16.958 & 0.008 & 1.199 & -4.921 & -8.864 & 1.293 \\
\hline 23 & ${ }_{73}^{156} \mathrm{Ta}$ & 2 & $3 / 2^{+}$ & $1028(5)$ & 22.384 & -0.050 & 1.292 & -0.620 & -1.433 & 1.448 \\
\hline 24 & ${ }_{73}^{156} \mathrm{Ta}^{*}$ & 5 & $11 / 2^{-}$ & $1130(8)$ & 21.350 & -0.050 & 1.278 & 0.949 & -3.099 & 1.177 \\
\hline 25 & ${ }_{73}^{157} \mathrm{Ta}$ & 0 & $1 / 2^{+}$ & $947(7)$ & 23.322 & 0.045 & 1.303 & -0.523 & -0.523 & 1.099 \\
\hline 26 & ${ }_{75}^{160} \mathrm{Re}$ & 2 & $3 / 2^{+}$ & $1284(6)$ & 20.587 & 0.080 & 1.261 & -3.046 & -3.837 & 1.649 \\
\hline 27 & ${ }_{75}^{161} \mathrm{Re}$ & 0 & $1 / 2^{+}$ & $1214(6)$ & 21.172 & 0.080 & 1.270 & -3.432 & -3.432 & 1.275 \\
\hline 28 & ${ }_{75}^{161} \mathrm{Re}^{*}$ & 5 & $11 / 2^{-}$ & $1338(6)$ & 20.167 & 0.080 & 1.254 & -0.488 & -4.433 & 1.626 \\
\hline 29 & ${ }_{77}^{164} \mathrm{Ir}$ & 5 & $11 / 2^{-}$ & $1844(9)$ & 17.644 & 0.089 & 1.201 & -3.959 & -7.768 & 1.838 \\
\hline 30 & ${ }_{77}^{165} \mathrm{Ir}$ & 5 & $11 / 2^{-}$ & $1733(7)$ & 18.201 & 0.099 & 1.212 & -3.456 & -7.279 & 1.594 \\
\hline 31 & ${ }_{77}^{166} \mathrm{Ir}^{*}$ & 5 & $11 / 2^{-}$ & $1340(8)$ & 20.699 & 0.107 & 1.257 & -0.076 & -3.955 & 1.578 \\
\hline 32 & ${ }_{77}^{167} \mathrm{Ir}$ & 0 & $1 / 2^{+}$ & $1086(6)$ & 22.993 & 0.116 & 1.289 & -0.959 & -0.959 & 1.492 \\
\hline 33 & ${ }_{77}^{167} \mathrm{Ir}^{*}$ & 5 & $11 / 2^{-}$ & $1261(7)$ & 21.338 & 0.116 & 1.266 & 0.875 & -3.012 & 1.670 \\
\hline 34 & ${ }_{79}^{171} \mathrm{Au}$ & 0 & $1 / 2^{+}$ & $1469(17)$ & 20.291 & -0.105 & 1.245 & -4.770 & -4.770 & 1.498 \\
\hline 35 & ${ }_{79}^{171} \mathrm{Au}^{*}$ & 5 & $11 / 2^{-}$ & $1718(6)$ & 18.763 & -0.105 & 1.217 & -2.654 & -6.414 & 1.901 \\
\hline 36 & ${ }_{81}^{177} \mathrm{Tl}$ & 0 & $1 / 2^{+}$ & $1180(20)$ & 23.223 & -0.050 & 1.282 & -1.174 & -1.174 & 1.342 \\
\hline 37 & ${ }_{81}^{177} \mathrm{Tl}^{*}$ & 5 & $11 / 2^{-}$ & $1986(10)$ & 17.901 & -0.053 & 1.192 & -3.347 & -7.006 & 2.648 \\
\hline 38 & ${ }_{83}^{185} \mathrm{Bi}$ & 0 & $1 / 2^{+}$ & $1624(16)$ & 20.293 & -0.052 & 1.232 & -4.229 & -4.229 & 2.436 \\
\hline
\end{tabular}



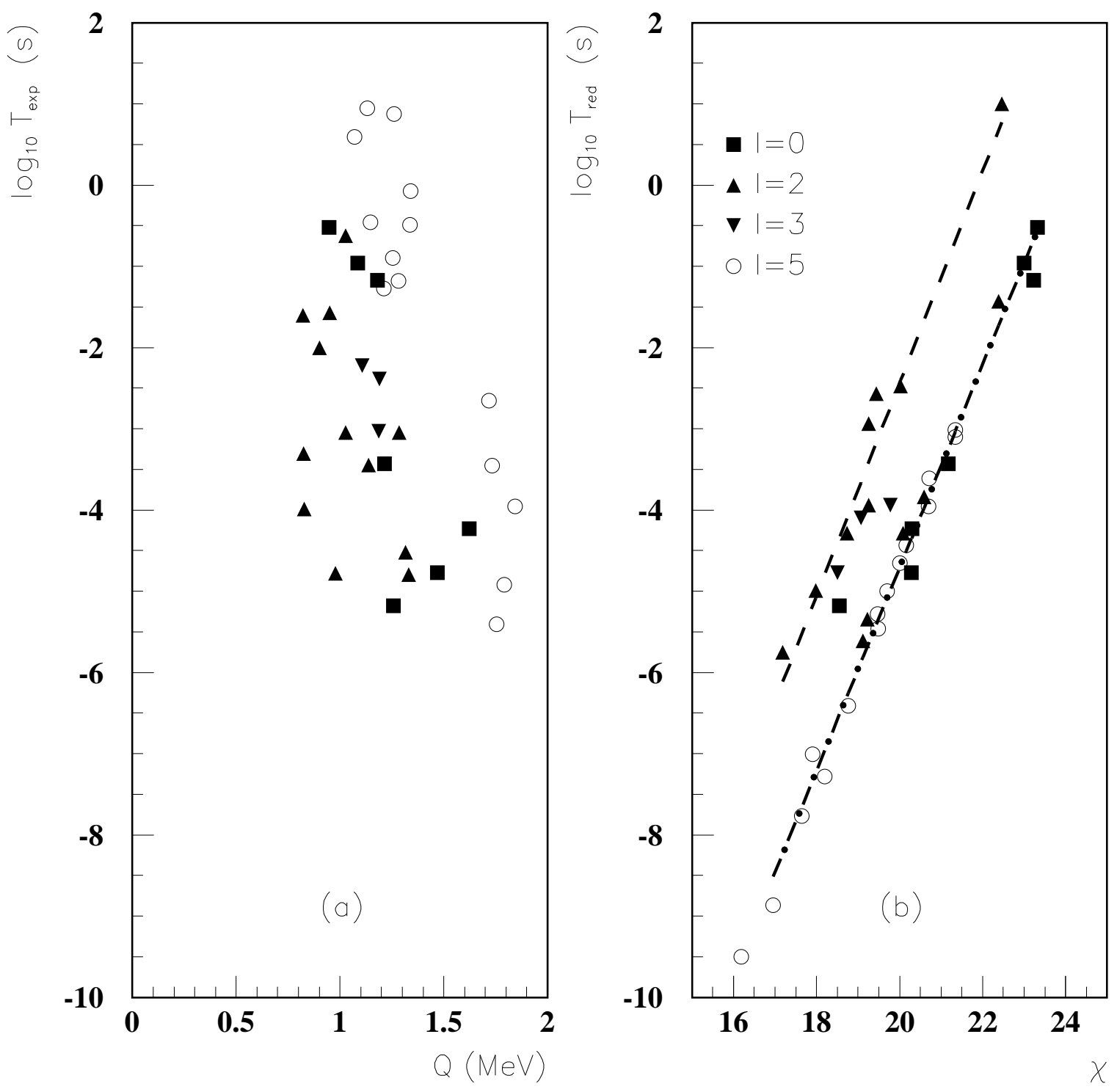

FIG. 1: (a) Logarithm of the experimental half-lives corresponding to proton decay as a function of the Q-value. The data are taken from Ref. [4].

(b) Values of $\log _{10} T_{r e d}$, Eq. (6), as a function of the Coulomb parameter $\chi$, The numbers labelling the different symbols correspond to the $l$-values of the outgoing proton. The two lines are computed according to Eq. (8). 


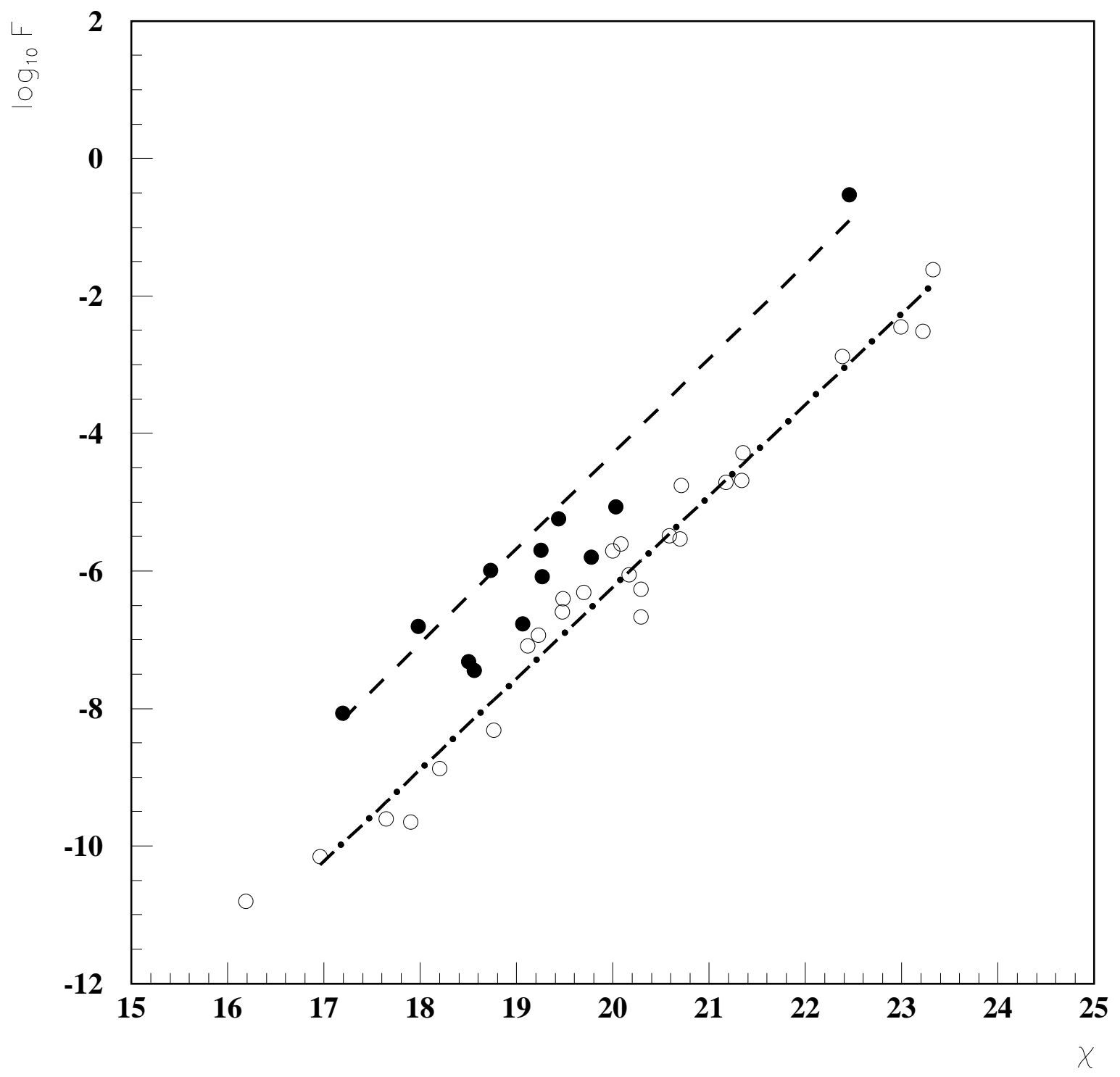

FIG. 2: The logaritm of $F(\chi, \rho)(\mathrm{s} / \mathrm{fm})$, Eq. (7), as a function of $\chi$. The two straight lines are average values to guide the eye. Filled symbols correspond to the first twelve cases of Table 1. 

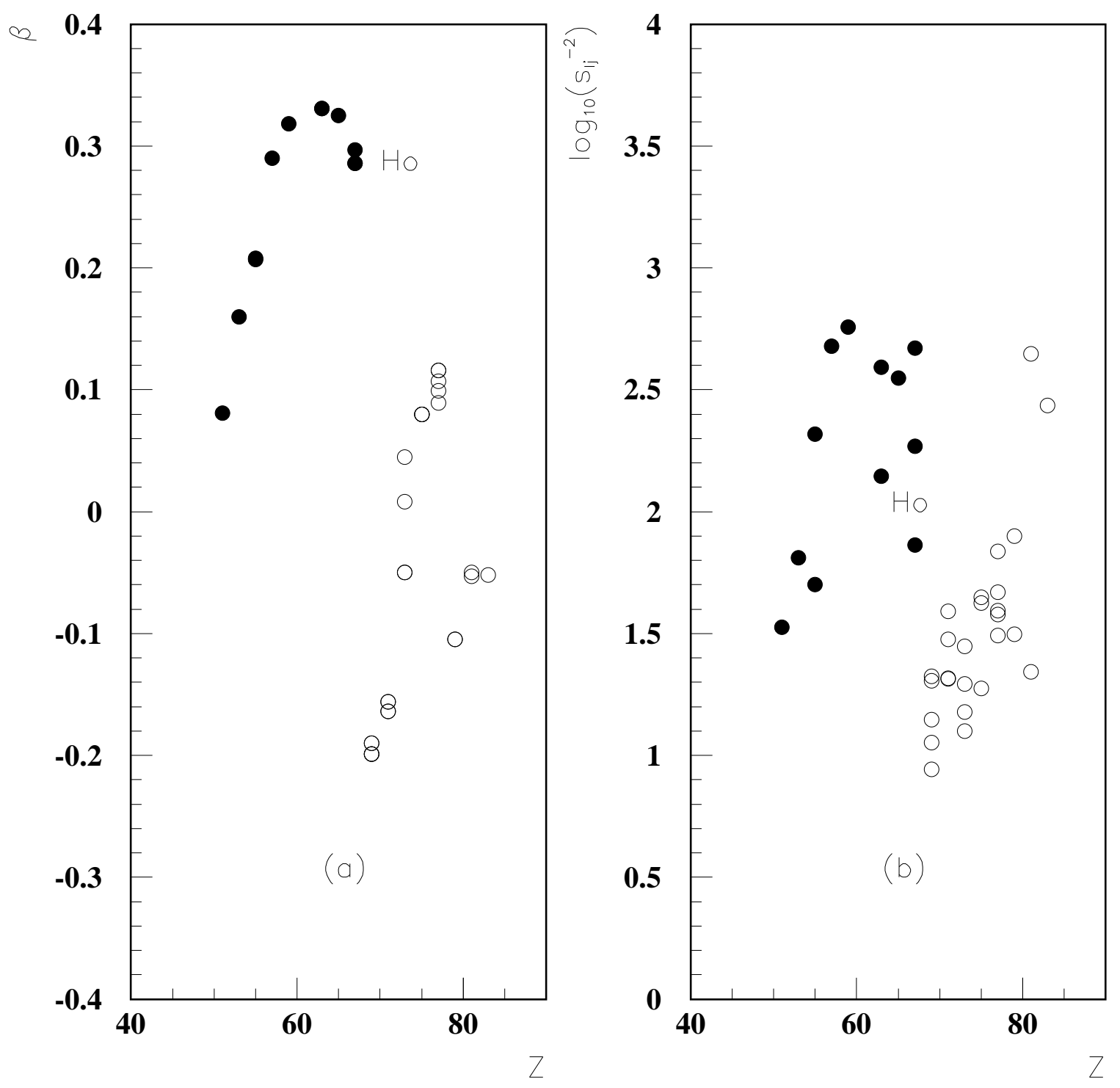

FIG. 3: (a) Dependence of the quadrupole deformation parameter $\beta$, as given in Table 1, upon the charge number Z. Filled symbols correspond to the first twelve cases of Table 1 . Notice that $\beta$ is the same in this scale for the isotopes $\mathrm{Cs}$ and also for the two states in $\mathrm{Ho}$.

(b) The logarithm of the spectroscopic function $s_{l j}^{-2}$, defined by Eq. (1) and given in the last column of the Table 1 , versus the charge number $\mathrm{Z}$. 